\title{
Research on coal-rock recognition based on sound signal analysis
}

\author{
Xihui Chen ${ }^{1,2}$ a, Zenan Yang ${ }^{1}$ and Gang Cheng ${ }^{2}$ \\ ${ }^{1}$ College of Mechanical and Electrical Engineering, Hohai University, Changzhou, 213022, China \\ ${ }^{2}$ School of Mechatronic Engineering, China University of Mining and Technology, Xuzhou, 221116, China
}

\begin{abstract}
The recognition of the cutting state of shearer is the key technology to realize variable speed cutting and mining automation. It is of great significance for improving shearer reliability, ensuring personal safety and improving coal quality. This paper proposed a coal-rock recognition method based on sound signal analysis. The original sound signal produced during the cutting process of shearer is decomposed by variational mode decomposition (VMD), and the obtained IMFs can construct a signal matrix. The signal matrix is processed by singular value decomposition (SVD), and a series of singular values can be obtained and defined as the signal features. Finally, the coal-rock recognition is realized by extreme learning machine (ELM) based on the extracted signal features. The experiment results show that the overall recognition accuracy is $91.7 \%$ under the actual cutting condition, which verifies the effectiveness of the proposed method in coal-rock recognition, and lays a theoretical foundation for the automation and intellectualization of shearer mining.
\end{abstract}

\section{Introduction}

The recognition of the cutting state of shearer is the key technology to realize variable speed cutting and mining automation. It is of great significance for improving shearer reliability, ensuring personal safety and improving coal quality. At present, there are many kinds of methods for coal-rock recognition, the main technical methods are force detection method, image processing detection method, ray detection method, vibration detection method and sound detection method and so on $[1,2]$. However, the force detection method has some defects of inaccurate force measurement. Because there is a lot of dust in the real working conditions of coal mine, so the effect of image processing detection method is well affected by external environmental interference. The ray detection method has a good effect, but the price of the sensors is high and the real application cost is high. The vibration signal generated by collision between coal and rock in shearer cutting process is different, so the method based on vibration signal detection is proposed. But the vibration should be reduced on the transmission path, and the feature should be weakened in its transmission process, which is not conducive to feature extraction [3]. The sound signal generated by the cutting of shearer also contains the information reflecting the cutting state of shearer. In addition, compared with vibration signals, the measurement of sound signals is belongs to non-contact measurement method, and the measurement of sound signals can effectively reduce the loss of signal transmission process [4]. Analyzing the sound signal generated during the cutting process of shearer is a feasible method to realize coal-rock recognition.

Similar to vibration signal analysis, the key of coalrock recognition based on sound signal is also feature extraction and state recognition. The sound signal generated by shearer cutting also has the characteristics with non-stationary and non-linear [5]. At present, nonstationary signal analysis method usually includes wavelet transform, empirical mode decomposition (EMD). But in the applied process of wavelet transform, it is very difficult to determine the most suitable wavelet basis. EMD also has some obvious drawbacks, such as modal aliasing [6]. To solve this problem, EEMD is proposed, but the decomposed components obtained by EEMD generally do not conform to the basis definition of IMF [7]. Recently, VMD is proposed, and it is adaptive and without time-frequency interference. VMD decomposes the original complex sound signal into a series of finite narrowband-limited IMFs by searching for the optimal solution of the variational constrained model, and the instantaneous frequency of the obtained IMFs has practical physical significance. At present, VMD has been applied to deal with non-stationary signals $[8,9]$.

A series of IMFs can be obtained by VMD, and the state information in the sound signals of shearer cutting can be stored among them. The effective features could be extracted from the signal matrix consisting of those IMFs. SVD is often used in signal processing and feature extraction, and the obtained singular values can mine the important information hidden in the signal matrix [10]. Therefore, SVD can be used to process the signal matrix

a Corresponding author: Xihui Chen; Email: chenxh@hhu.edu.cn 
composed of a series of IMFs, and the obtained singular values can be used as the signal feature to reflect the state difference among signals. State recognition is the key after signal feature extraction. ELM is a singlehidden layer feed-forward neural network, and it has been widely applied in many fields due to its good learning ability. This algorithm randomly generates the connection weights between input layer and hidden layer and the threshold of hidden neurons, and they do not need to be adjusted in the training process. The number of hidden neurons is the only parameter that need to be set up, and the unique optimal solution can be obtained. Compared with other traditional training methods, ELM has the advantages of fast learning and good generalization ability [11, 12]. Based on the above analysis, a coal-rock recognition method based on sound signal analysis is established, which combines VMD, SVD and ELM.

\section{Model establishment}

\subsection{Variational mode decomposition (VMD)}

The decomposition process of VMD is the solution process of variational problems. The algorithm can be divided into the construction and solution of variational problems.

1. The construction of variational problem

Assuming that each modal function has the finite bandwidth with a central frequency, the variational problem can be described as finding $K$ modal functions $u_{k}(t)$, and the sum of the estimated bandwidth of each modal function is the smallest. The constraint condition is the sum of each modal function is the original signal, and the specific construction is as follows $[13,14]$ :

(1) The analytical signal of each modal function $u_{k}(t)$ is obtained by Hilbert transform, and its unilateral spectrum can be obtained;

(2) The frequency spectrum of each modal function is modulated to the corresponding baseband by mixing the estimated centre frequency;

(3) The L2 norm of the gradient square of the above demodulation signal is calculated, and the bandwidth of each modal signal can be estimated.

Assuming that the original signal $s(t)$ is decomposed into $K$ IMFs $u_{k}(t)$, the corresponding constrained variational model is as follows:

$$
\left\{\begin{array}{l}
\min _{\left\{u_{k}\right\},\left\{\omega_{k}\right\}}\left\{\sum_{k}\left\|\partial_{t}\left[\left(\delta(t)+\frac{j}{\pi t}\right) u_{k}(t)\right] \mathrm{e}^{-j \omega_{k} t}\right\|\right\} \\
\text { s.t. } \quad \sum_{k} u_{k}=s(t)
\end{array}\right.
$$

where $\left\{u_{k}\right\}$ is the decomposed $K$ IMFs, and $\left\{u_{k}\right\}=\left\{u_{1}, u_{2}, \ldots, u_{K}\right\} ;\left\{\omega_{k}\right\}$ is frequency center for each IMF, and $\left\{\omega_{k}\right\}=\left\{\omega_{1}, \omega_{2}, \ldots, \omega_{K}\right\}$.

2. The construction of variational problem

(1) The quadratic penalty factor $\alpha$ and Lagrange multiplication operator $\lambda(t)$ are introduced, and the constrained variational problem is transformed into a non-constrained variational problem, and the augmented Lagrange expression is as follows:

$$
\begin{aligned}
& L\left(\left\{u_{k}\right\},\left\{\omega_{k}\right\}, \lambda\right)=\alpha \sum_{k}\left\|\partial_{t}\left[\left(\delta(t)+\frac{j}{\pi t}\right) u_{k}(t)\right] \mathrm{e}^{-j \omega_{k} t}\right\|^{2} \\
& +\left\|s(t)-\sum_{k} u_{k}(t)\right\|^{2}+\left\langle\lambda(t), s(t)-\sum_{k} u_{k}(t)\right\rangle
\end{aligned}
$$

(2) Alternate direction method of multipliers is applied to solve the variational problems, in VMD, and the saddle point of Lagrange expression can be obtained by altering $u_{k}^{n+1}, \omega_{k}^{n+1}$ and $\lambda_{k}^{n+1}$. Where the value of $u_{k}^{n+1}$ can be expressed as [8]:

$$
u_{k}^{n+1}=\arg \min \left\{\begin{array}{l}
\alpha\left\|\partial_{t}\left[\left(\left(\delta(t)+\frac{j}{\pi t}\right) * u_{k}(t)\right)\right] \mathrm{e}^{-j \omega_{k} t}\right\|^{2} \\
+\left\|s(t)-\sum_{i} u_{i}(t)+\frac{\lambda(t)}{2}\right\|_{2}^{2}
\end{array}\right\}
$$

Where $\omega_{k}$ is equivalent to $\omega_{k}^{n+1}, \sum u_{i}(t)$ is equivalent to $\sum_{i \neq k} u_{i}(t)^{n+1}$, and Eq. (3) is converted to frequency domain.

$$
\hat{u}_{k}^{n+1}=\underset{\hat{u}_{k}, u_{k} \in X}{\arg \min }\left\{\begin{array}{l}
\alpha\left\|j \omega\left[\left(1+\operatorname{sgn}\left(\omega+\omega_{k}\right)\right) \hat{u}_{k}\left(\omega+\omega_{k}\right)\right]\right\|_{2}^{2} \\
+\left\|\hat{s}(t)-\sum_{i} \hat{u}_{i}(\omega)+\frac{\hat{\lambda}(\omega)}{2}\right\|_{2}^{2}
\end{array}\right\}
$$

Further $\omega-\omega_{k}$ is used to replace $\omega$ of first item.

$$
\hat{u}_{k}^{n+1}=\underset{\hat{u}_{k}, u_{k} \in X}{\arg \min }\left\{\begin{array}{l}
\alpha\left\|j\left(\omega-\omega_{k}\right)\left[(1+\operatorname{sgn}(\omega)) \hat{u}_{k}(\omega)\right]\right\|_{2}^{2} \\
+\left\|\hat{s}(t)-\sum_{i} \hat{u}_{i}(\omega)+\frac{\hat{\lambda}(\omega)}{2}\right\|_{2}^{2}
\end{array}\right\}
$$

Next, Eq. (5) is transformed into the form of interval integral with non negative frequency, and the solution of the two optimization problem can be expressed as follows:

$$
\hat{u}_{k}^{n+1}(\omega)=\frac{\hat{s}(\omega)-\sum_{i \neq k} \hat{u}_{i}(\omega)+\frac{\hat{\lambda}(\omega)}{2}}{1+2 \alpha\left(\omega-\omega_{k}\right)^{2}}
$$

According to the same process, the value problem of central frequency is converted to frequency domain:

$$
\omega_{k}^{n+1}(\omega)=\underset{\omega_{k}}{\arg \min }\left\{\int_{0}^{\infty}\left(\omega-\omega_{k}\right)^{2}\left|\hat{u}_{k}(\omega)\right|^{2} d \omega\right\}
$$

The central frequency update method is as follows:

$$
\omega_{k}^{n+1}=\frac{\int_{0}^{\infty} \omega\left|\hat{u}_{k}(\omega)\right|^{2} d \omega}{\int_{0}^{\infty}\left|\hat{u}_{k}(\omega)\right|^{2} d \omega}
$$

where $\hat{u}_{k}(\omega)$ is equivalent to the Wiener filtering of the remaining amount $\hat{s}_{k}(\omega)-\sum_{i \neq k} \hat{u}_{i}(\omega) . \omega_{k}^{n+1}$ is the gravity of power spectrum of current modal function, and the real part of the inverse Fourier transform of $\left\{\hat{u}_{k}(\omega)\right\}$ is $\left\{u_{k}(t)\right\}$.

3. Algorithm process

Step1: $\left\{\hat{u}_{k}\right\},\left\{\omega_{k}^{1}\right\},\{\hat{\lambda}\}$ and $n$ are initialized;

Step2: $u_{k}$ and $\omega_{k}$ are updated according to Eq. (6) and Eq. (8);

Step3: $\lambda$ is updated according to follows: 


$$
\hat{\lambda}^{n+1}(\omega) \leftarrow \hat{\lambda}^{n}(\omega)+\tau\left(\hat{s}(\omega)-\sum_{k} \hat{u}_{k}^{n+1}(\omega)\right)
$$

Step4: For given discriminant accuracy $e>0$, if $\sum_{k}\left\|\hat{u}_{k}^{n+1}-\hat{u}_{k}^{n}\right\|_{2}^{2} /\left\|\hat{u}_{k}^{n}\right\|_{2}^{2}<e$, the iteration is stopped. Otherwise, go to step 2 for execution.

\subsection{Singular value decomposition (SVD)}

All IMFs obtained by VMD can construct a signal matrix. SVD is used to process this signal matrix, and the obtained singular values are defined as the signal feature. The process of SVD is as follows:

Assuming that the obtained IMFs by VMD are expressed as $\left\{u_{1}(t), u_{2}(t), \ldots, u_{k}(t)\right\}$, and those IMFs can construct a signal, and it is expressed as follows:

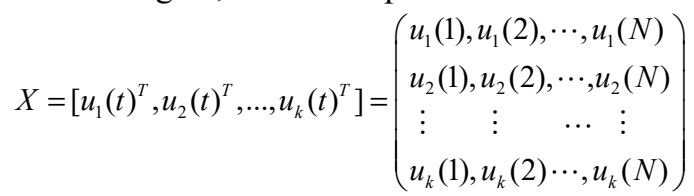

where $u_{k}(t)$ is the $k$-th IMF, and $N$ is the length of IMF.

For the signal matrix in Eq. (10), SVD is carried out, and $X=U D V^{T} . U$ and $V$ are normalization matrix, and $D$ is the diagonal matrix constructed by the obtained singular value $\sigma_{i}$, where $\sigma_{i}=\sqrt{K \lambda_{i}}$, and $\lambda_{i}$ correspond to the eigenvalues of covariance matrix $C=X X^{T} / K$ of matrix $X[15]$.

Through the above process, singular values $\sigma_{1}, \sigma_{2}, \ldots, \sigma_{K}$ can be obtained and used as the features extracted from the signal matrix.

\subsection{Extreme learning machine (ELM)}

The input layer, hidden layer and output layer of ELM are connected by weight matrix, and the connection weights between input layer and hidden layer and the connection weights between hidden layer and output layer are expressed as follows $[11,16]$ :

$$
w=\left[\begin{array}{cccc}
w_{11} & w_{12} & \ldots & w_{1 h} \\
w_{21} & w_{22} & \ldots & w_{2 h} \\
\vdots & \vdots & & \vdots \\
w_{J 1} & w_{J 2} & \ldots & w_{J h}
\end{array}\right], \beta=\left[\begin{array}{cccc}
\beta_{11} & \beta_{12} & \ldots & \beta_{1 k} \\
\beta_{21} & \beta_{22} & \ldots & \beta_{2 k} \\
\vdots & \vdots & & \vdots \\
\beta_{H 1} & \beta_{H 2} & \ldots & \beta_{H k}
\end{array}\right]
$$

The threshold value of hidden neurons is as follows:

$$
\phi=\left[\phi_{1}, \phi_{2}, \ldots, \phi_{H}\right]^{T}
$$

Assuming that there is $Q$ training samples, and each sample corresponds to an output vector, and the input matrix and output matrix of the samples are expressed as follows:

$x i=\left[\begin{array}{cccc}x i_{11} & x i_{12} & \ldots & x i_{1 Q} \\ x i_{21} & x i_{22} & \ldots & x i_{2 Q} \\ \vdots & \vdots & & \vdots \\ x i_{J 1} & x i_{J 2} & \ldots & x i_{J Q}\end{array}\right]_{J \times Q} \quad, y o=\left[\begin{array}{cccc}y o_{11} & y o_{12} & \ldots & y o_{1 Q} \\ y o_{21} & y o_{22} & \ldots & y o_{2 Q} \\ \vdots & \vdots & & \vdots \\ y o_{K 1} & y o_{K 2} & \ldots & y o_{K Q}\end{array}\right]_{K \times Q}$

Assuming that the activation function of the hidden neurons is $f(x)$, and the output $T$ of ELM is as follows:

$$
T=\left[t_{1}, t_{2}, \ldots, t_{Q}\right]_{K \times Q}=\left[\begin{array}{c}
\sum_{i=1}^{H} \beta_{i 1} f\left(w_{i} x i_{h}+\phi_{i}\right) \\
\left.\sum_{h}^{H} \beta_{1 h}, t_{2 h}, \ldots, t_{K h}\right]_{1 \times K}^{T}=\left[\begin{array}{c}
\left.x_{i} x i_{h}+\phi_{i}\right) \\
\vdots \\
\sum_{i=1}^{H} \beta_{i K} f\left(w_{i} x i_{h}+\phi_{i}\right)
\end{array}\right]_{K \times 1} \quad(h=1,2, \ldots, Q)
\end{array}\right.
$$

where $w_{i}=\left[w_{i 1}, w_{i 2}, \ldots, w_{i J},\right]$, and $x i_{h}=\left[x i_{1 h}, x i_{2 h}, \ldots, x i_{J h},\right]^{T}$.

Eq. (14) can be simplified as follows:

$$
F \beta=T^{*}
$$

where $T^{*}$ is the transpose of $T, F$ is the output matrix of the hidden layer of ELM.

According to ELM theory $[12,16]$, only some parameters of ELM need to be adjusted when the activation function $f(x)$ of hidden neuron is infinitely differentiable. The connection weights $w$ between input layer and hidden layer and the threshold $\phi$ of hidden layer can be generated randomly when the initial network is established, and they remain unchanged during ELM training process. The connection weights $\beta$ between hidden layer and output layer can be obtained by calculating the least squares solution.

$$
\min _{\beta}=\left\|F \beta-T^{*}\right\|
$$

The training of ELM can be completed when the output matrix of the hidden layer of ELM is determined, and the trained ELM can be used to complete the recognition task.

\section{Sound signal acquisition experiment}

The cutting experiment of coal mine was carried out, the sound sensor in cutting experiment is shown in Figure 1. The sound signals collected from the rocker arm of MG900/2400-WD shearer are obtained. The shearer runs at $2 \mathrm{~m} / \mathrm{min}$ speed when cuts coal and rock, and the sampling frequency is $44 \mathrm{kHz}$. The experimental scene of underground coal mine is shown Figure 2.
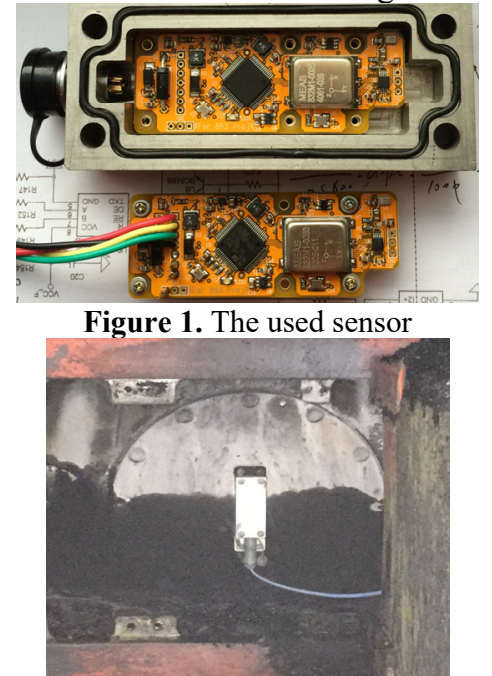

Figure 2. The experimental scene of underground coal mine. 


\section{Experimental analysis}

The sound signals of two kinds of cutting states including cutting coal and cutting rock can be collected by experiment, and the sound signals are shown in Figure 3 . The preliminary analysis of the sound signals shows that the cutting state of shearer can not be distinguished by the time-domain waveform of the sound signals.

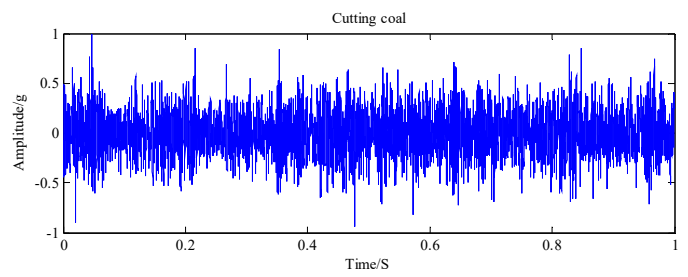

Cutting rock

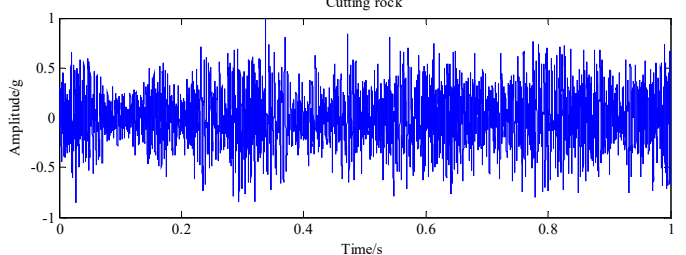

Figure 3. The sound signals of two kinds of cutting states.

Next, VMD is used to process the sound signals, and the final number of the decomposed IMFs is set as 8 . The VMD results of the sound signals for cutting coal and cutting rock are shown in Figure 4 and Figure 5, respectively.
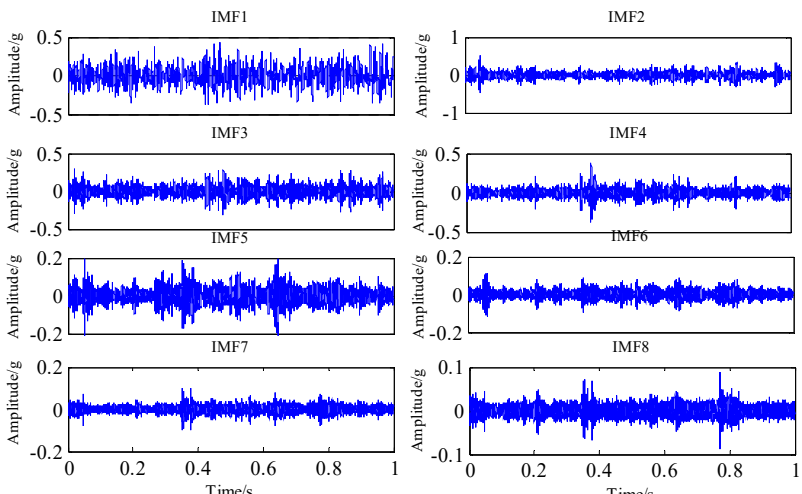

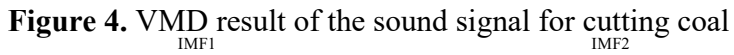
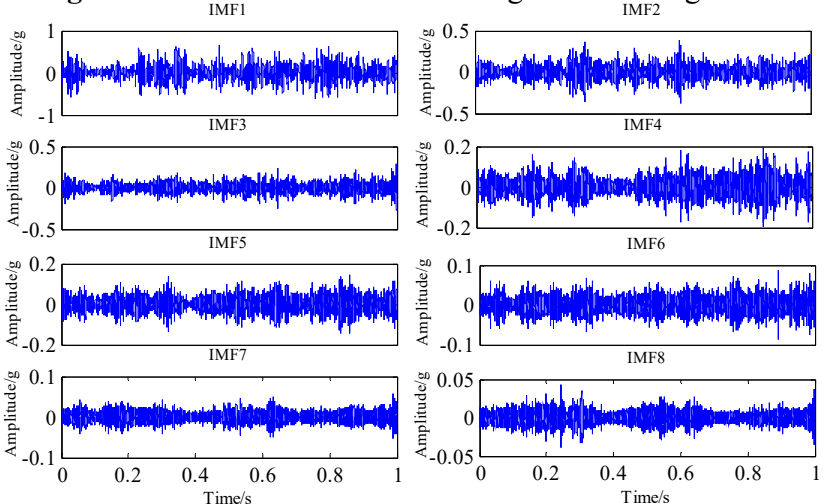

Figure 5. VMD result of the sound signal for cutting rock

It can be seen from Figure 4 and Figure 5 that the sound signals of cutting coal and cutting rock are decomposed into 8 IMFs by VMD. There are some salient points in each IMF, and comparing the VMD results of sound signals of cutting coal and cutting rock, it can be seen that the amplitudes of IMFs are different.
Although the IMF amplitudes between cutting coal and cutting rock are somewhat different (E.g. IMF1 and IMF5), the amplitude difference of IMF is not enough to accurately recognize the current cutting state. But each IMF contains some feature information, and all IMFs can construct a signal matrix. Next, state feature extraction is performed, and in this paper, the state feature is the singular values of the signal matrix constructed by IMFs. Limited by the paper space, the extracted state features of 6 sound signal samples of cutting coal and cutting rock are shown in Figure 6. It can be seen that the state features of sound signal of cutting rock are generally greater than those of cutting coal. Although there are some fluctuations in the state features of different sound samples under the same cutting state, the fluctuations are within an acceptable range. Meanwhile, they will still interfere with the state recognition. Next, ELM is used for coal-rock recognition.

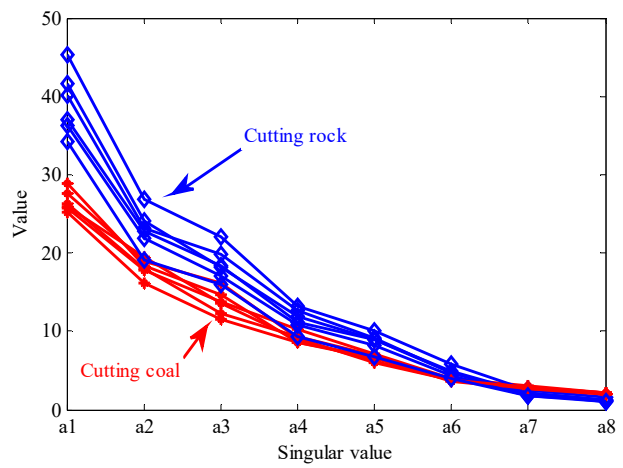

Figure 6. The state features of cutting coal and cutting rock.

30 training sound samples are prepared for each cutting state, and a total of 60 training sound samples. Each sample is processed to extract the state features as the input of ELM, and ELM has 8 input neurons. After many experiments, the activation function of hidden neurons is selected to sig function, and the number of hidden neurons is selected to 18 . When the training of ELM is completed, the trained ELM can be used to complete the coal-rock recognition task. Similarly, 30 testing samples are randomly selected from each cutting state and recognized by the trained ELM. The output of the trained ELM is shown in Figure 7, and the recognition rate of cutting state is shown in Table 1.

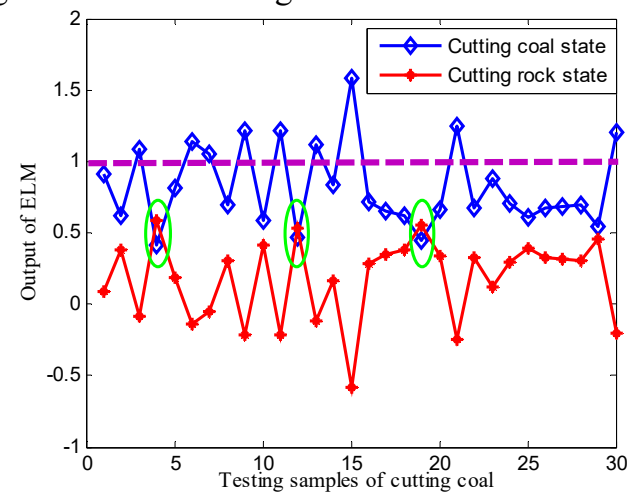

(a) Testing samples of cutting coal 


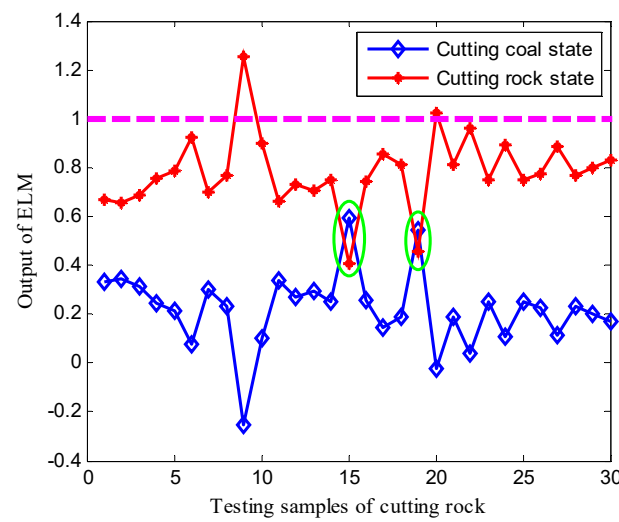

(b) testing samples of cutting rock

Figure 7. The output of the trained ELM

Table 1. Recognition rate of cutting state

\begin{tabular}{cccc}
\hline $\begin{array}{c}\text { Cutting } \\
\text { state }\end{array}$ & $\begin{array}{c}\text { Cutting } \\
\text { coal }\end{array}$ & $\begin{array}{c}\text { Cutting } \\
\text { rock }\end{array}$ & $\begin{array}{c}\text { Overall } \\
\text { recognition rate }\end{array}$ \\
\hline $\begin{array}{c}\text { Recognition } \\
\text { rate }\end{array}$ & $90 \%$ & $93.3 \%$ & $91.7 \%$ \\
\hline
\end{tabular}

It can be seen from Figure 7 and Table 1 that 3 of 30 sound testing samples of cutting coal are misrecognized as cutting rock, and 2 of 30 sound testing samples of cutting rock are misrecognized as cutting coal. The recognition rate of cutting coal is $90 \%$, and the recognition rate of cutting rock is $93.3 \%$. The proposed method is an effective method for coal-rock recognition.

\section{Calculations}

In this paper, the sound signal generated in the cutting process of shearer is taken as the research object, and the research of coal-rock recognition method is developed. VMD is used to process the original sound signal, and it can decompose the sound signal with non-stationary and non-linear into $8 \mathrm{IMFs}$. The state information is also included in each IMF, and these IMFs constitute a signal matrix. Singular values extracted from the signal matrix can quantitatively represent the cutting state information, and the state features of sound signal of cutting rock are generally greater than those of cutting coal. The extracted state features are defined as the input of ELM, and the coal-rock state can be effectively recognized by the trained ELM. By experiment, it can be proved that the recognition rate of cutting coal is $90 \%$, and the recognition rate of cutting rock is $93.3 \%$. The proposed coal-rock recognition method which combines VMD, SVD and ELM is an effective method.

\section{Acknowledgment}

This work was supported by the Fundamental Research Funds for the Central Universities (Grant number 2017B07914), this support is gratefully acknowledged.

\section{References}

1. J.P. Sun, B. Chen. Journal of China Coal Society, 40, S2 (2015)

2. J.P. Sun. Coal Science Technology, 39, 9 (2011)
3. [3] B.P. Wang, Z.C. Wang, W.Z. Zhang. Journal of Vibration Measurement \& Diagnosis, 32, 4 (2012)

4. G.X. Zhang, Z.C. Wang, L. Zhao. Shock and Vibration, 3809525 (2017)

5. J. Xu, Z.B. Wang, C. Tan, L. Si, X.H. Liu. Sensors, 18, 2 (2018)

6. N.E. Huang, Z. Shen, S.R. Long.Proceedings of the Royal Society A, 454, (1998)

7. J. Xu, Z.B. Wang, C. Tan, L. Si, X.H. Liu. Sensors, 15, 11 (2015)

8. M. Sahani, P.K. Dash. Neurocomputing, 310, (2018)

9. H.S. Xiao, J.C. Wei, H.S. Liu. IET Generation Transmission \& Distribution, 11, 16 (2017)

10. T.K. Araghi, A. Abd Manaf, S.K. Araghi. Expert Systems with Application, 112, (2018)

11. X.H. Chen, G. Cheng, H.Y. Li, Y. Li. Strojniški vestnik - Journal of Mechanical Engineering, 63, 1 (2017)

12. G.B. Huang, Q.Y. Zhu, C.K. Siew. Neurocomputing, 70, (2006)

13. Y.X. Li, Y. Li, X. Chen. Symmetry-Basel, 9, 11 (2017)

14. X. Zhang, Q. Miao, H. Zhang. Mechanical Systems and Signal Processing, 108, (2018)

15. O.I. Traore, L. Pantera, N. Favretto-Cristini, P. Cristini, S. Viguier, P. Vieu. Measurement, 104, (2017)

16. G.B. Huang, L. Chen, C.K. Siew. IEEE Transactions on Neural Networks, 17, 4 (2006) 